\title{
“Leukoplakia- Potentially Malignant Disorder of Oral Cavity -a Review"
}

\author{
Neha Aggarwal*1 and Sumit Bhateja ${ }^{2}$ \\ ${ }^{1}$ Department of Oral Medicine \& Radiology, Manav Rachna Dental College \& Hospital, Faridabad, India \\ ${ }^{2}$ Reader Dept of Oral Medicine and Radiology, Manav Rachna Dental College, India
}

Received: May 18, 2018; Published: May 29, 2018

*Corresponding author: Neha Aggarwal, Senior Lecturer (MDS), Department of Oral Medicine \& Radiology, Manav Rachna Dental College \& Hospital, Faridabad, India

\begin{abstract}
The term Leukoplakia simply means a "white patch", and it has been used in a sense to describe any white lesion in the mouth. This non-specific usage led to confusion among physician, surgeons and researchers who attributed a precancerous nature to many innocuous lesions. Some investigators tried, although unsuccessfully, to restrict this term only to those white lesions that histologically indicated epithelial dysplasia. Since the mid-1960s there has been a considerable understanding and clarification in the concept of leukoplakia, and now leukoplakia is recognized as a specific entity.
\end{abstract}

Keywords: Leukoplakia; Potentially malignant disorder

\section{Introduction}

Leukoplakia is a greek word- Leucos means white and Plakiameans patch. It was first coined in the second half of the 19th century by the Hungarian dermatologist, Schwimmer in 1877 [1,2]. WHO (1978) [3]- A white patch or plaque that cannot be characterized clinically or pathologically as any other disease. This definition does not carry any histologic connotation. Over a 25 year period the WHO definition for leukoplakia has been quoted by researchers and clinicians alike, and adapted or refined by other working groups and experts at several international seminars. International attempts to define/refine the WHO definition of oral leukoplakia are:

First International Conference on Oral Leukoplakia Malmo, Sweden (1983) [4] -A white patch or plaque that cannot be characterized clinically or pathologically as any other disease and is not associated with any physical or chemical causative agent except the use of tobacco [4].

International Symposium, Uppsala, Sweden (1996) [5] - A predominantly white lesion of the oral mucosa that cannot be characterized as any other definable disease. WHO (1997) [6] - A predominantly white lesion of the oral mucosa that cannot be characterized as any other definable lesion. Warnakulasuriya et al. [2] - Leukoplakia should be used to recognize white plaques of questionable risk having excluded (other) known diseases or disorders that carry no increased risk for cancer. Leukoplakia is a clinical term and the le sion has no specific histology. It may show atrophy or hyperplasia (acanthosis) and may or may not demonstrate epithelial dysplasia. It has a variable behavioural pattern but with an assessable tendency to malignant transformation.

\section{Epidemiology}

The prevalence of leukoplakia in India varies from $0.2 \%$ to $4.9 \%$. Men are affected more frequently than women, and a vast majority of leukoplakia occurs in the age range of 35-45 years. Less than $1.3 \%$ of leukoplakias in India are idiopathic.1Leukoplakia is seen most frequently in middle-aged and older men, with an increasing prevalence with age [7]. Less than one percent of men below the age of 30 have leukoplakia, but the prevalence increases to an alarming eight percent in men over the age of 70 . The prevalence in women past the age of 70 is approximately two percent. The most common sites are the buccal mucosa, alveolar mucosa, and lower lip; however, lesions in the floor of mouth, lateral tongue, and lower lip are most likely to show dysplastic or malignant changes [8].

\section{Classification}

\section{According to BANOCZY (1977) [2]}

A. Type I - Leukoplakia Simplex- auniformraised plaque formation, varying in size, with regular edges. 
B. Type II - LeukoplakiaVerrucosa - a lesionwithslightlyraised, rounded, red or white excrescence, thatmaybedescribed as granules or nodules.

C. Type III - Leukoplakia Erosiva- it is characterized by verrucous proliferation raised above the mucosal surface.

\section{According to WHO 1980 [3]}

Homogenous leukoplakia - Lesion that was uniformly white and unscrapable. Non-homogenous leukoplakia - Lesion predominantly white and speckled with red.

\section{PAPE et al (1994) [2]}

A. Homogenous: It is completely whitish lesion.

i. Flat- It has smooth surface.

ii. Corrugated- like a beach at ebbing edge.

iii. Pumice like- with a pattern of fine lines

iv. Wrinkled - like dry, cracked mud surface.

B. Non- Homogenous: Proliferative \& Verrucous- slow growing, papillary proliferations, above the mucosal surface that may be heavily keratinized. Ulcerated- Lesion exhibits red area at the periphery of which white patches are present. Nodular - Characterized by white specks or nodules on erythematous base. Erythroleukoplakia - leukoplakia is present in association with erythroplakia.

\section{According to WHO (1998) [3]}

Thin, smooth leukoplakia- Translucent thin gray soft flat plaques usually with sharply demarcated borders. Thick, fissured leukoplakia- 2/3 of white plaques has distinctly white appearance, fissured and is leathery to palpation. Granular, verruciformleukoplakia- Lesions have surface irregularities of nodular or granular nature with verrucous appearance. Erythroleukoplakia- Lesion showing intermixed red and white areas. According to WHO (2002) depending on the probability of a malignant change and prognosis of these lesions as

a. Phase I: thin, smooth leukoplakia - better prognosis.

b. Phase II: thick, fissured leukoplakia.

c. Phase III: proliferative verrucousleukoplakia (PVL) - higher malignant transformation rate.

d. Phase IV: erythroleukoplakia - poor prognosis

\section{WARNAKULASURIYA et al (2007) [3]}

Homogeneous leukoplakia

Non - Homogenous leukoplakia

Speckled leukoplakia

Nodular leukoplakia

Verrucousleukoplakia

\section{Staging System [3]}

A clinical staging system for oral leukoplakia (OL system) on the lines of TNM staging was recommended by WHO in 2005 taking into account the size (L) and the histopathological features (P) of the lesion. (L - Size of leukoplakia)

L1 -Size of leukoplakia is $<2 \mathrm{~cm}$

L2 - Size of leukoplakia is 2 - $4 \mathrm{~cm}$

L3 - Size of leukoplakia is $>4 \mathrm{~cm}$

Lx - Size of leukoplakia is not specified

I. (P - Pathology)

Px - Dysplasia not specified in pathology report

P0 - No epithelial dysplasia

P1 - Mild to moderate epithelial dysplasia

P2 - Severe epithelial dysplasia

\section{OLEP Staging System}

\author{
Stage I L1P0 \\ Stage II L2P0 \\ Stage III L3P0 or L1/L2P1 \\ Stage IV L3P1 or any LP2.
}

\section{General Rules of the OLEP Staging System}

If there is doubt concerning the correct $\mathrm{L}$ or $\mathrm{P}$ category to which a particular case should be allotted, than the lower (i.e. less advanced) category should be chosen. This will also be reflected in the stage grouping. In case of multiple biopsies of single leukoplakia or biopsies taken from multiple leukoplakias the highest pathological score of the various biopsies should be used. Leukoplakia is purely a clinical terminology and histopathologically it is reported as epithelial dysplasia. WHO in 2005 proposed five grades of epithelial dysplasia based on architectural disturbances and cytological atypia.
a. Squamous Hyperplasia - benign lesion.
b. Mild Dysplasia - better prognosis.
c. Moderate Dysplasia.
d. Severe Dysplasia.
e. Carcinoma In-situ - poor prognosis.

It has been recently proposed to modify the above 5- tier system into a binary system of 'high risk' and 'low risk' lesions to improve clinical management of these lesions.

\section{Etiopathogenesis $[9,10]$}

Local Factors: TOBACCO - It is the main etiologic agent for leukoplakia. It is available in two forms: smoked and smokeless. The smoked form contains carbon monoxide, thiocyanate, hydrogen 
cyanide, nicotine and the metabolites of these constituents whereas smokeless tobacco contains nitrosamine, polycylic aromatic hydrocarbons and nitrosoproline. The smoked tobacco is available in the forms of bidi, chilum and cigarette whereas the smokeless tobacco is available in the forms of dry snuff, moist snuff, niswar, naas, mishri, khaini quid (tobacco + slaked lime). The chemical constituents of tobacco and its combustion end products as tars and resins are irritating substances capable of causing leukoplakia. Over 300 carcinogens have been identified in tobacco smoke or in its water-soluble components which can be expected to leach into saliva. The major and most studied among them include aromatic hydrocarbons, benzopyrene and the tobacco specific nitrosamines, N-nitrosonornicotine (NNN), nitrosopyrrolidine (NYPR), nitrosodimethylamine (NDMA) and 4-(methylnitrosamine)-1-(3-pyridyl)1-butanone (NNK).Benzopyrene is a powerful carcinogen and is found in amounts of $20-40 \mathrm{mg}$ per cigarette. The mainstream smokes of a cigarette contains $310 \mathrm{mg}$ of NNN and $150 \mathrm{ng}$ of NNK. These agents act on keratinocytes, stem cells and are absorbed and act in many other tissues in the body.

Alcohol: It seems to have a strong synergistic effect with tobacco relative to oral cancer production, has not been associated with leukoplakia. People who excessively use mouth rinses with alcohol content greater than $25 \%$ may have grayish buccal mucosal plaques, but these are not true leukoplakia. Alcohol causes dehydration of the oral mucosa and increases the ambient temperature of the oral cavity thereby making the oral mucosa more vulnerable to the carcinogenic effects of tobacco. Alcohol by itself contains known hydrocarbons and nitosamines.

Sanguinaria: This is a herbal extract used in the toothpaste and mouth rinse. It can cause true leukoplakia. This type of leukoplakia is called sanguinaria-associated keratosis and is usually located in the maxillary vestibule or on the alveolar mucosa of the maxilla.

Trauma: Continuous trauma or local irritation in the oral cavity is suspected as a causative agent for leukoplakia. The source of irritation may be malocclusion, ill fitting denture, sharp broken teeth, hot or spicy food, root piece, etc. The usual site to such irritation is the buccal mucosa and less often is the alveolar ridge. Chronic mechanical irritation can produce a white lesion with a roughened keratotic surface termed frictional keratosis.

Candidiasis: The presence of Candida albicans has been reported very frequently in association with leukoplakia, more commonly with nodular type. Candidalleukoplakiamay be associated with other local factors such as tobacco smoking, denture wearing or occlusal friction. Tobacco smoking may result in candidal colonization because of increased keratinization, reduced salivary IgA concentration or decreased PMNL function. There is a long standing discussion whether candida infection is a cause of leukoplakia or if it is a superimposed infection in a pre-existing lesion. It has been shown that, upon treatment, non-homogenous candida-infected leukoplakia convert into a homogenous lesion, and some lesions even regressed.

\section{Regional \& Systemic Factors:}

\section{i. Tertiary Syphilis}

White patches are seen on the tongue. Syphilitic glossitis is observed. Atrophy of the filiform and fungiform papillae occurs.

\section{Deficiency of vitamin A,B complex, C,E beta-carotene}

a. Nutritional Deficiency: Sideropenicanemia may be the disposing factor for the occurrence of leukoplakia.

b. Viral Infection: The possible implication of human papilloma virus in the etiology and potential for the malignant transformation of oral premalignant lesion has been studied extensively and it was reported that the likelihood of detecting HPV was 2-3 times higher in precancerous oral mucosa and 4-5 times higher in squamous cell carcinoma than in normal oral epithelium. The possible viral etiology of oral leukoplakia had been first suggested by light microscopic examination of HPV suggestive changes. In a follow up study of 20 leukoplakias; Lind (1987) established a significant correlation between the prescence of HPV antigen and the degree of dysplasia and malignant transformation. Studies by Nielson (1996) in the prevalence of HPV in oral premalignant lesions found an overall rate of HPV positive lesions to be $40.8 \%$ of which five of them were HPV 16. Laryngeal squamous cell papilloma and recurrent respiratory papillomatosis (RRP) are well-established HPV-induced tumours.

c. Idiopathic Leukoplakia: In a small proportion of cases, no underlying cause has been found. Such lesions are called idiopathic leukoplakia. They have higher potential for malignant transformation.

\section{d. Clinical Features [9]}

A. Leukoplakia usually affects persons older than 40 years of age. Prevalence increases rapidly with age, especially for males and as many as $8 \%$ of men older than 70 years of age reportedly are affected.

B. Leukoplakia may be found in almost any location in the oral cavity, it is more prevalent of the buccal mucosa, gingiva and vermilion border of the lip (actinic cheilitis). The lips and palate, maxillary mucosa, retromolar area, floor of the mouth and tongue are less likely sites. Two latter sites account for $93 \%$ of leukoplakia with dysplasia or carcinomatous change.

C. Sex distribution is variable. Men are more affected in some countries, while this is not the case in the western world. Less than $1 \%$ of men below the age of 30 have leukoplakia. The male -to-female ratio is reported to be about 3:1 to $6: 1$.

D. Leukoplakia can be either solitary or multiple.

E. Early or thin leukoplakia appears as a slightly elevated grayish-white plaque that may be either well-defined or may gradually blend into the surrounding normal mucosa. As the lesion progresses, it becomes thicker and whiter, sometimes 
developing a leathery appearance with surface fissures. Some leukoplakias develop surface irregularities and are referred to as granular or nodular leukoplakias. Other lesions develop a papillary surface and are known as verrucous or verruciformleukoplakia.

\section{Clinical Appearance: $[1,10]$}

a. Homogenous Leukoplakia: It refers to a localized lesion or extensive white patch that present a relatively consistent pattern throughout. Surface of lesion is described variously as corrugated ("like a beach at ebbing tide") with a pattern of fine lines ("cristae"), wrinkled ("like dry, cracked mud") or papillomatous. About $84 \%$ of the leukoplakias are of the homogeneous type.

b. Non-homogenous Leukoplakia: Nodular leukoplakia-Nodular leukoplakia (also called speckled leukoplakia) is characterized by small white specks or nodules on an erythematous base, the nodules may be very fine (Speckled), pinhead sized or even larger. About 3\% of leukoplakias are of the nodular type. It is associated with higher malignant transformation rate.

Verrucousleukoplakia: It is a term used to describe the presence of thick white lesions with papillary surfaces in the oral cavity. These lesions are heavily keratinized and are most often seen in older adults in the 6th to 8th decades of life.

Proliferative Verrucous Leukoplakia: Proliferative verrucousleukoplakia (PVL) is a unique type of clinical oral leukoplakia. Enigmatic in etiology, PVL behaves in a far more aggressive fashion than other forms of leukoplakia. Batsakis JG et al. (1999) reported its aggressiveness relates not only to a high recurrence rate, but more so to a very high level of and relentless progression from a localized simple keratosis to extensive oral disease and squamous carcinomas of verrucous or conventional squamous cell type. Diagnosis is often late in the protracted course of PVL with the disease in an advanced stage when it is especially refractory to treatment. Campisia G, Giovannellib L et al. (2004) reported that proliferative verrucousleukoplakia (PVL) is a very aggressive form of oral leukoplakia (OL) with high morbidity and mortality rates, hypothesized to be linked to HPV infection. Among the different clinical varieties of leukoplakias, PVL has got the highest risk of malignancy. In a follow up study of 54 cases of PVL, Silverman and Gorsky found that $70.3 \%$ of the patients subsequently developed squamous cell carcinoma [9].

Candidal Leukoplakia: Candidalleukoplakia is an extremely chronic form of oral candidiasis which is seen as firm, white, leathery plaques and are found on buccal mucosa, lips and tongue. Differentiation of candidalleukoplakia from other forms of leukoplakia is based on PAS staining for hyphae and antibody studies. Epithelial dysplasia occurs 4 to 5 times more frequently in candidalleukoplakia than in leukoplakia in general. Carcinomatous change, which occurs up to $40 \%$ more frequently in candidalleukoplakia, is a characteristic of the speckled lesions.

Malignant Transformation: LeukoplakiaTranformation Risk by Anatomical Site. (Figure 1) In one large, well known retrospective study that looked at approximately 3,300 biopsies of oral white lesions, Waldron and Shafer determined that 19.9 percent of leuko- plakias showed some degree of epithelial dysplasia. In this group, 3.1 percent were unsuspected squamous cell carcinoma, 4.6 percent showed severe dysplasia or carcinoma in situ, and 12.2 percent showed mild-to-moderate epithelial dysplasia. The location of oral leukoplakia has a significant correlation with the frequency of finding dysplastic or malignant changes at biopsy. In the study by Waldron and Shafer, the floor of mouth was the highest-risk site, with 42.9 percent of leukoplakias showing some degree of epithelial dysplasia, carcinoma in situ, or unsuspected invasive squamous cell carcinoma [8]. The clinical appearance of leukoplakia may also indicate some correlation with the likelihood that the lesion will show dysplastic or malignant features. In general, the thicker the leukoplakia, the greater the chance of finding dysplastic changes; therefore, a verrucousleukoplakia is more likely to show dysplasia than is a thick homogeneous leukoplakia, which, in turn, is more likely to show dysplasia than is a thin leukoplakia. The frequency of dysplastic or malignant alterations in oral leukoplakia has ranged from 15.6 to $39.2 \%$ in several studies. A wide range of rates for the malignant transformation of leukoplakia has been reported from 0.13 to $19.8 \%$ [8].

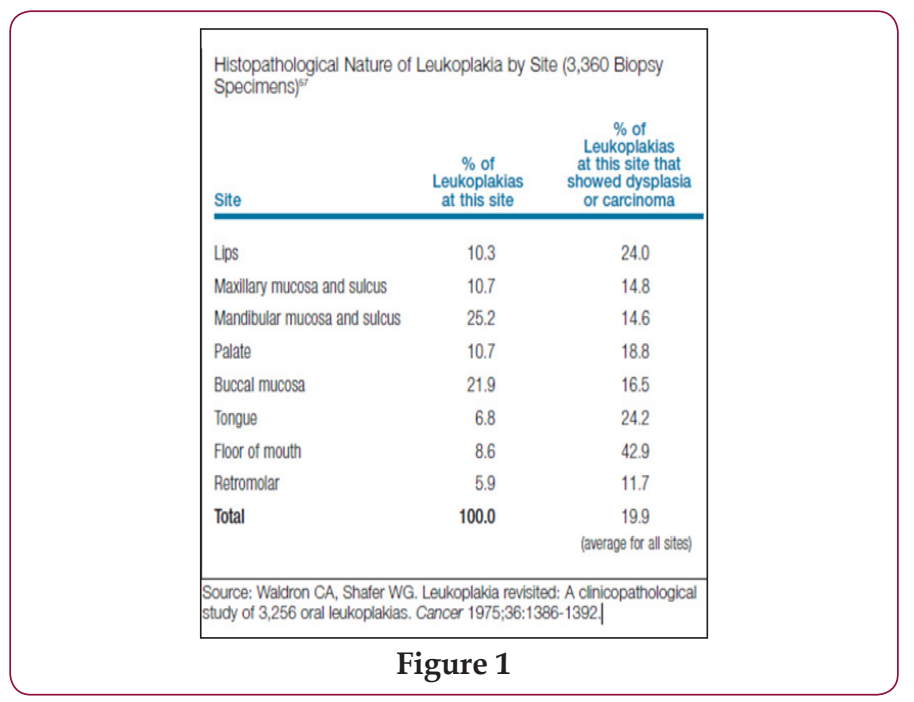

\section{Diagnosis}

A provisional diagnosis of leukoplakia is made when a predominantly white lesion at clinical examination cannot be clearly diagnosed as any other disease or disorder of the oral mucosa. So, a biopsy is mandatory. The various diagnostic aids used are as follows:

\section{Clinical Methods: [10]}
A. Vital Staining
i. Toluidine Blue
ii. Lugol's Iodine Vizilite

\section{Photodiagnosis}

i. 5-Aminolevulinic acid mediated fluorescence endoscopic imaging

ii. 5-Aminolevulinic acid mediated digitized fluorescence endoscopic imaging

iii. Autofluorescence spectroscopy 


\section{Histopathological Methods/Cytological Methods}

i. Exfoliative cytology

ii. Oral CDx system

iii. Biopsy

\section{Clinical Methods}

\section{Toluidine Blue [10]}

Toluidine blue is an acidophilic metachromatic dye which selectively stains acidic tissue components. Applying a 1\% aqueous solution of toluidine blue to the lesion, rinsing with $1 \%$ solution of acetic acid, rinsing with water and then observe for any binding. The accuracy has been found to exceed $90 \%$. The probable mechanism is the affinity or binding of toluidine blue with DNA and sulfatedmucopolysaccharides, both of which are selectively high in dysplastic and malignant oral epithelium compared with normal epithelium and benign lesions. Additionally, toluidine blue dye binds to cytoplasmic negatively charged mitochondrial membranes, which are stronger in dysplastic and malignant epithelial cells compared with normal tissue. The method has good sensitivity with a very low false negative rate. The dorsum of the tongue always stains positively, due to the retention of the dye in the crevice between the papillae. It is effective in demonstrating dysplasia and early malignant lesion which is not clinically recognizable.

\section{Mechanism}

Binding with DNA- It is an acidophilic, metachromatic nuclear dye of thiazine group that selectively stains acidic tissue components particularly nucleic acid such as DNA and RNA. Dysplastic and anaplastic areas which contains more DNA than normal cells. Intracellular canal - Malignant epithelium contains intracellular canals that are wider than normal epithelium which also facilitates the penetration of dye. Effect on normal epithelium- Most of the epithelial surfaces stain blue after the application of $1 \%$ toluidine blue solution but the stain is lost after application of $1 \%$. Acetic acid solution to normal epithelium surface.

\section{Contents}
i. Toluidine blue- $1 \mathrm{gm}$
ii. Acetic acid $-10 \mathrm{cc}$
iii. Absolute alcohol $-4.19 \mathrm{cc}$
iv. Distilled water- $86 \mathrm{cc}$
v. $\quad$ PH adjusted to 4.5

\section{Technique}
a. Rinsing the mouth with water
b. Ask patient to rinse with $1 \%$ acetic acid.
c. Dry the area with gauze
d. Apply $1 \%$ toluidine blue solution to the lesion
e. Ask the patient to rinse again with acetic acid and then with water.

\section{Lugol's Iodine}

Richart used lugol's iodine for delineation of malignant change. The normal tissue is stained brown and proliferating epithelium is unstained or poorly stained. This solution produces a brown black stain by reaction of iodine with glycogen. It is retained in normal squamous epithelial cells but not in dysplastic or malignant cells of the squamous epithelium. In the oral tissues, Lugol's iodine has less sensitivity in identifying oral premalignant and dysplatic diseases. But, is of greater specificity.

\section{Vizilite4}

Vizilite is based on the principle of chemiluminescence comprising of a chemiluminescent light source to improve the identification of lesions and a dye to mark those lesions identified by vizilite. Vizilite passes over oral tissue that has been treated with the rinse solution, normal healthy tissue will absorb the light and appear dark, abnormal tissue will appear white. Vizilite kit consists of a Vizilite 1\% acetic acid solution, a capsule or a lightstick. The vizilite capsule or chemiluminescent light stick comprises an outer flexible plastic capsule containing aspirin or acetyl salicylic acid and an inner fragile glass vial containing hydrogen peroxide. Activation of the capsule is achieved by flexing it wherein, the inner fragile glass vial ruptures releasing the hydrogen peroxide. The chemicals react to release the energy which excite the electrons in the fluorescent dye and converts this energy into the light of the white-blue color with a wavelength ranging from $430-580 \mathrm{~nm}$. The light lasts for approximately 10 minutes \& is long enough to do a thorough visual oral examination.

\section{Photodiagnosis}

\section{5-Aminolevulinic Acid mediated Fluorescence Endo- scopic Imaging}

After oral administration or topical application of aminolevulinic acid and the synthesis of protoporphyrin within the dysplastic cells, these cells can be easily detected by the fluorescence of protoporphyrin.

\section{5-Aminolevulinic Acid Mediated Digitized Fluorescence Endoscopic Imaging}

This technique developed has the capability of acquiring high quality on line images and quantifying the fluorescence intensity of diseased oral tissues. It can be a useful adjunct to pathological diagnosis, for directing biopsies and assessing resection margins for oral cavity lesions.

\section{Autofluorescence Spectroscopy}

It is applicable for the detection of alterations in the structural composition of cells which indicate the presence of diseased tissue. It is due to several endogenous fluorophores and can be useful in guiding the optimal location of biopsy.

\section{Histopathological Methods / Cytological Methods [11] Exfoliative Cytology}

It is the microscopic examination of shed cells from an epithelial surface. Its application in diagnosis of premalignant lesions is limited due to the occurrence of false negative results, because many 
lesions have a thick keratinized surface layer and contain subtle changes of dysplasia that may be difficult to detect in a smear.

a. Thecytologic Smear is Reported by the Cytologist into five Classes:

i. Normal - Indicates only normal cells.

ii. Atypical - Indicates the presence of minor atypia but no malignant changes.

iii. Indeterminate - The cells display wider atypia suggestive of premalignant lesions. Biopsy is recommended.

iv. Suggestive of dysplasia - Few cells with malignant characteristics or many cells with borderline characteristics. Biopsy is must.

v. Positive for dysplasia - Obviously malignant cells. Biopsy is must.

Scanning electron microscopic examination of the surface cells from both erosive and verrucousleukoplakias has revealed the presence of surface irregularities to these cells that are associated with premalignant \& malignant changes as detected by conventional light microscopic examination of tissue sections.

\section{b. Oral CDx System [10]:}

Oral brush biopsy is a development that is been heavily marketed to dentists and was introduced in 1999. Oral brush cytology uses a special brush to collect the epithelial cells. The technique uses a round stiff bristle brush to collect cells from the surface and subsurface layers of a lesion by vigorous abrasion. The brush is placed in contact with oral epithelium and rotated with firm pressure 5 to 10 times.

i. If the Technique is Properly Performed, the Brush Collects Cells from all three Layers of the Epithelium Namely:
A. The basal layer
B. The intermediate layer
C. The superficial layer

That's why this technique is also known as transepithelial biopsy because it collects the cells from all the three layers. The cellular material collected on the brush is transferred to a glass slide and flooded with fixative ( alcohol or propylene glycol) If the sample is adequate, a computer that is specifically programmed to detect oral epithelial dysplastic and malignant cells scan each brush cytology specimen and displayed on a high resolution color video monitor.

ii. The Pathologist Classifies the Oral brush Cytology Specimen into one of three Categories:

a. Negative - No epithelial abnormality is detected.

b. Positive - Definitive cellular evidence of epithelial dysplasia or carcinoma is present.

c. Atypical - Abnormal epithelial changes are present.

\section{Biopsy [12]}

Biopsy is the surgical removal of a tissue from a living person for microscopic examination to confirm or to establish the diagnosis of a disease. It can be excisional biopsy, incisional biopsy or punch biopsy. Excisional biopsy is the total excision of the small lesion (less than $1 \mathrm{~cm}$ in size) for the histopathological examination. Incisional biopsy is the removal of a small, representative portion of the lesion for microscopic examination, if area under investigation appears difficult to excise because of its extensive size (i.e. larger than $1 \mathrm{~cm}$ in diameter). Punch biopsy is a form of incisional biopsy in the oral cavity. It is best suited for the diagnosis of mucosal abnormalities that may require multiple biopsies. It was first described by Lynchand Morris. Keyes biopsy punches are the type recommended for use on oral mucosal lesions. Most important in the diagnosis of oral leukoplakia is the determination of cellular dysplasia by microscopy. Benign forms are characterized by hyperkeratosis, chronic inflammatory cell infiltration in corium. Dysplasia is characterized by abnormal orientation of epithelial cells, cellular pleomorphism, cellularatypia suggestive of early malignancy.

\section{Grading of Dysplasia \\ Epithelial Dysplasia6}

i. Drop-shaped rete ridges

ii. Nuclear hyperchromatism

iii. Cellular pleomorphism and altered nuclear cytoplasmic ratio

iv. Excess mitotic activity

v. Loss of polarity of basal cells

vi. Deep cell keratinisation

vii. Disordered or loss of differentiation

viii. Loss of intercellular adherence

\section{Mild Dysplasia}

In this lesion there is a thin layer of parakeratin and the structure, maturation and orderly differentiation of the epithelial cells is largely unaffected. However, there is a degree of irregularity of basal cells with variation in size and hyperchromatism.

\section{Moderate Dysplasia}

The dermal papillae extend close to the surface and there are elongated rete processes, some of which are broader deeply. Enlarged and hyperchromatic cells are visible at this low power in rete processes and in most of the prickle cell layer.

\section{Severe Dysplasia}

This rete process is composed almost entirely of cells with dark and irregularly shaped nuclei. Only the most superficial layers of cells show maturation to squamous cells and the orderly maturation and differentiation of epithelial cells has been lost (Figure 2). Clinical observation of the suspected leukoplakia without the biopsy/ follow up is dangerous. The clinical response of hyperkeratotic 
areas of oral mucosa is unpredictable and rebiopsy at 6-12 month intervals is indicated, particularly if the lesion changes in size or physical characteristics. Somewhere between $6 \%$ and $10 \%$ of leukoplakia with evidence of dysplasia progress to malignancy. Except for a limited number of lesions and locations, the clinical features of leukoplakic lesions in general are a poor predictor of which will become malignant. Because of the higher risk that nodular (speckled) and verrucousleukoplakia and leukoplakia of the floor of mouth and tongue dorsum will become malignant, excision of these lesions with adequate follow up is very much essential. Recurrence will often follow removal of the lesion if recognized irritants and predisposing factors are not eliminated.

\section{Treatment Options}
a. Cessation of tobacco use and observation
b. Antifungal agents (Candida-associated leukoplakia)
c. Chemoprevention- Retinoids; Vitamins (A, C, E); Carote noids
d. Topical therapy- Bleomycin; Vitamin A
e. Photodynamic therapy
f. Surgical excision with or without grafting
g. Electrocoagulation
h. Cryosurgery
i. $\quad$ Laser surgery

\section{Role of Retinoids in Leukoplakia}

Retinoids are the natural and synthetic derivatives of vitamin A. Epidemiological studies indicate that a low intake of vitamin A is associated with an increased risk of squamous cancer. In vitro studies on cancer cells show that exposure to retinoids results in the inhibition of growth by blocking the cell cycle or by inducing apoptosis. With respect to the clinical efficacy of retinoids some positive effects have been observed in early stages of oral and or opharyngeal cancer. Administration of retinoids has been shown to elicit responses in leukoplakia, a premalignant lesion of the oral mucosa that frequently develops into invasive cancer [13].

\section{Role of oral Lycopene in Leukoplakia}

Mohitpal Singh, R. Krishanappa et al. [14] conducted a study and observed effect of lycopene and suggested that it can be effectively and safely used for the management of oral leukoplakia. Lycopene, the carotenoid that gives the ripe tomato its bright red color, is a very effective natural antioxidant and quencher of free radicals. It is also found in various fruits such as watermelons, guava and pink grapefruit. Reactive oxygen species (ROS) is generated in tissues and can damage DNA, proteins, carbohydrates and lipids. Lycopene exhibits the Results indicate that lycopene can protect cells against cell damage and play a protective role against progression of dysplasia [14]. Dose of $8 \mathrm{mg} /$ day is beneficial in the treatment of oral leukoplakia [9].

\section{Role of Beta- Carotene in Leukoplakia}

Beta-Carotene acts as a chemopreventive agent for oral leukoplakia and studies reported it as the best chemopreventive agent in comparison with retinol or alpha - tocopherol that have also been recommended for clinical use against oral precancer. In order to raise the serum levels of beta carotene, increased consumption of yellow/green and orange vegetables or fruits may thus be recommended as a primary preventive measure against oral cancer. Beta carotene increases the number of circulating lymphocytes, enhances the proliferation and induction of cytotoxic T cells, increases the number of helper $\mathrm{T}$ cells, increases tumor necrosis factor and enables natural killer cells to be more effective. Ingestion of beta carotene quickly increases the number of CD4 helper T lymphocytes and increases the $\mathrm{T}$ lymphocytes response to mitogens. So, it enhances the immune response.

\section{Role of Topical Bleomycin in Leukoplakia [15]}

A study was conducted to confirm the effectiveness of topical bleomycin treatment for leukoplakia. Results showed that an adequate concentration was required to maximize the benefit. A concentration of $1.0 \%$ was superior to $0.5 \%$, resulting in a complete response in three of five patients and a partial response in the remaining two.

\section{Role of Photodynamic Therapy in Leukoplakia}

Gluckman (1986) and Grant (1993) used additionally, a combined parentral and locally applied treatment modality in the form of photodynamic therapy. Systemic administration of anhematoporphyrin compound is followed by photo-irradiation with a specific form of laser energy by surface illumination.

\section{Various Surgical Treatment Procedures for Oral Leuko- plakia have been Reported}

\author{
i. Surgical excision with or without grafting \\ ii. Electrocoagulation \\ iii. Cryosurgery \\ iv. Laser surgery
}

Electrocoagulation produces thermal damage in the underlying tissue which causes postoperative pain, edema and leads to considerable scarring. Both postoperative pain and edema are also marked in cryosurgery. On the other hand, less postoperative pain and edema were reported in laser surgery in many previous studies.

\section{The Various Types of Lasers used for the Treatment of} Leukoplakia are: $[16,17]$
a. Carbon dioxide laser
b. Argon laser
c. Nd: YAG laser
d. KTP laser (Potassium titanyl phosphate) 


\section{The Mechanisms Involved are Laser Ablation or Laser Vaporization Advantages}

I. Laser surgery has a hemostatic effect, so it is very suitable for the surgical treatment.

II. It produces satisfactory mobility of oral mucosa after surgery and shows a very suitable postoperative oral function because of the limited scarring and cicatricial contraction.

III. It can be repeatedly performed, so it is favorable for the management of oral leukoplakia because lesions often recur.

IV. The thermal damage to adjacent tissue is minimal and it produces a good quality of life for the patients by reducing postoperative pain, swelling, edema and infection.

\section{Disadvantages:}

VI. A biopsy must be obtained by other methods either preoperatively or at the time of treatment with laser vaporization.

VII. The epithelial regeneration is delayed and wounds take longer to re- epithelialize than following excision surgery with sutures and small granuloma may sometimes be formed on the wound surface during healing.

VIII. Safety precautions as the use of eye- glasses are required to protect both the patient and the operator.

\section{References}

1. Mehta FS, Hammer JE (1992) Tobacco habits in India. Tobacco-related oral mucosal lesions and conditions in India. TIFR Bombay 8: 95

2. Garcia M, Jemal A, Ward EM, Center MM, Hao Y, Siegel RL, et al. (2007) Global Cancer Facts \&Figures 2007. American Cancer Society.

3. Neville BW, Day TA (2002) Oral Cancer and Precancerous Lesions. CA Cancer J Clin52: 195-215.

4. Axell T, Holmstrup P, Kramer IRH, J J Pindborg, M Shear (1984) International seminar on oral leukoplakia and associated lesions related to tobacco habits. Community Dent Oral Epidemiology 12:145-154.
5. Pindborg JJ, Reichart PA, Smith CJ, Vander Waal I (1997) World Health Organization International Histological Classification of Tumours. Histological typing of cancer and precancer of the oral mucosa. Berlin: Springer.

6. Rajendran R Benign, Malignant Tumors of the Oral Cavity (2009) In: Rajendran R, Sivapathasundaram B. Shafer's Textbook of Oral Pathology.6th ed. Elsevier pp: 80-218

7. Greenberg M, Glick M, Ship J (2002) Burket's Oral Medicine.9thed. BCDecker.

8. Waldron CA, Shafer WG (1975) Leukoplakia revisited. A clinicopathological study of 3256 oral leukoplakias. Cancer 36(4): 13861392.

9. Ongole R, BN Parveen (2013) Textbook of Oral Medicine Oral Diagnosis and Oral Radiology. Red and white lesions. Chennai, India: Elsevier $\left(2^{\text {nd }}\right.$ edn,). pp: 133-173.

10. Greenberg MS, Glick M (2005) Burket's Oral Medicine. Red and white lesions of the oral cavity. Delhi, India: BC decker Inc. Elsevier; $\left(10^{\text {th }} \mathrm{edn}\right.$.), pp. 85-125.

11. Banoczy J, Csiba A (1976) Occurence of epithelial dysplasia in oral leukoplakia. Oral Surg Oral Med Oral Patho 42: 766-774.

12. Greenberg MS, GlickM, Ship JA (2008) Burket's Oral Medicine. Red and white lesions of the oral cavity. BC Decker Inc, Hamilton: Elsevier; $\left(11^{\text {th }}\right.$ edn.), pp. 77-10613.

13. Sciubba JJ (1995) Oralleukoplakia. Vrit Rev Oral Biol Med 6(2): 147-160.

14. Singh M, Krishanappa R, Bagewadi A, Keluskar V (2004) Efficacy of oral lycopene in the treatment of oral leukoplakia. Oral Oncology 40: 591596.

15. Malmstrom M, Hietanen J, Sane J, Sysmalainen M (1988) Tropical treatment of oral leukoplakia with bleomycin.Br J Oral Maxillofacial Surg 26: 491-498.

16. Ishii J, Fujita K, Komori T (2003) Laser surgery as a treatment for oral leukoplakia. Oral Oncology 39: 759-769.

17. Reichart PA, Philipsen HP (2005) Oralerythroplakia-A review. Oral oncology 41: 551-561.

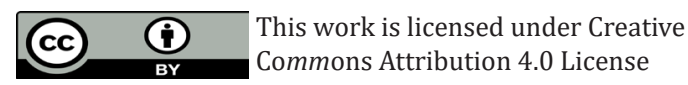

Submission Link: https://biomedres.us/submit-manuscript.php

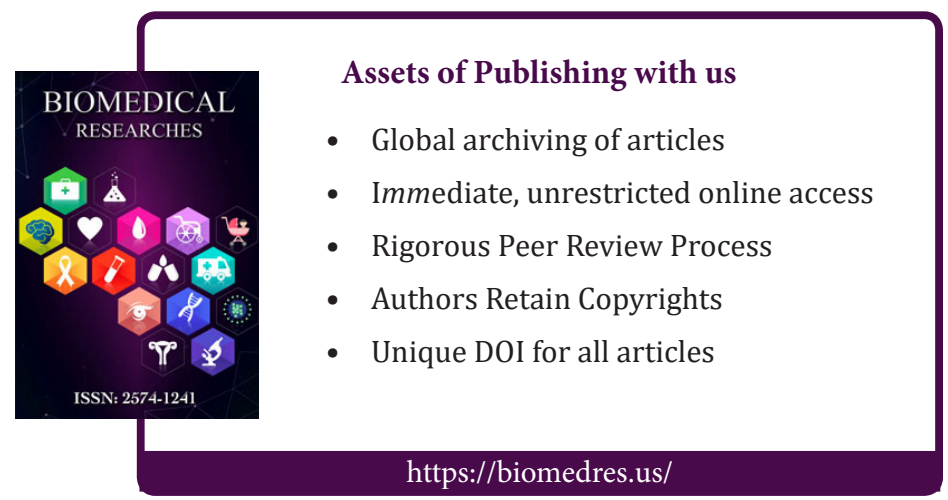

